\title{
Milligram per 24 Hours
}

National Cancer Institute

\section{Source}

National Cancer Institute. Milligram per 24 Hours. NCI Thesaurus. Code C67399.

A unit of mass flow rate equivalent to the rate at which one thousandth of a gram of matter crosses a given surface or is delivered to a given object or space over a period of time equal to twenty four hours. Milligram per twenty four hours is also a dose administration rate unit equal to the rate at which one thousandth of a gram of a product is administered per unit of time equal to twenty four hours. 